\title{
Epidemiological Study of Rapidly Emerging Uropathogens Isolated from Urinary Catheter and Its Influential Demographic Factors Responsible for Contamination
}

\author{
Anjali A. Tiwari*, Niraj Ghnawate, Yogesh Khalshinge \\ Department of Microbiology, Sant Gadge Baba Amravati University, Amravati, India \\ Email: *anjalitiwari2988@gmail.com
}

How to cite this paper: Tiwari, A.A., Ghnawate, N. and Khalshinge, Y. (2020) Epidemiological Study of Rapidly Emerging Uropathogens Isolated from Urinary Catheter and Its Influential Demographic Factors Responsible for Contamination. Advances in Microbiology, 10, 713-729.

https://doi.org/10.4236/aim.2020.1012051

Received: November 11, 2020

Accepted: December 28, 2020

Published: December 31, 2020

Copyright $\odot 2020$ by author(s) and Scientific Research Publishing Inc. This work is licensed under the Creative Commons Attribution International License (CC BY 4.0).

http://creativecommons.org/licenses/by/4.0/

\begin{abstract}
Urinary tract infections (UTI) are very common in all the developed as well as developing countries in which the majority of infections are catheter associated. Catheter associated urinary tract infections (CAUTI) is one of the major causes of hospital acquired infections. The aim of this study is to investigate influential demographic factors responsible for contamination associated with the rate of CAUTI, while taking into account type of urinary catheter used, the most common organisms found, patient diagnosis, age, gender, and comparison with other studies. During the study, 22 uropathogenic species were isolated from the different segments of urinary catheter samples of the patients collected from 12 different hospitals of Amravati city, Maharashtra, India from January 2015 to 2018. Gram negative organisms were the most frequent isolates, with Pseudomonas aeruginosa (16.08\%) being the most common followed by Escherichia coli (10\%) and Candida albicans accounted for almost $11 \%$ of all the uropathogens. It was found that the majority of uropathogens were isolated from the section A (Catheter segment inside the bladder) and section $\mathrm{E}$ (Catheter segment connected to drainage tube) of the urinary catheter. The duration of the catheterization plays the major role in the contamination and further infection to continue. It was observed that the female catheterized patients are more prone to the contamination and infection as compared to male catheterized patient. The antibiotic sensitivity pattern indicates that MAR (Multiple Antibiotic Resistance) index was more than 0.2 for almost all the uropathogens tested concluding that there is antibiotic stress on uropathogens and rate of resistance increased rapidly. Also it was found that there was a statistically significant association between the duration of catheterization, type of disease, age of patient and type of catheter with respect to gender.
\end{abstract}




\section{Keywords}

CAUTI, Uropathogens, Urinary Tract Infection, Urinary Catheter, Contamination

\section{Introduction}

Urinary tract infections (UTIs) are common, recurring diseases that are moderate to life-threatening. In addition to our growing understanding of the dangerous consequences conferred by the broad spectrum antibiotics use, the persistent emergence of antibiotic resistance gets increasing rapidly and UTI is the major cause of all catheter associated urinary tract infections. UTIs are recurrent infections that are precipitated primarily by UroPathogenic Escherichia coli (UPEC) bacteria that target about $80 \%$ of UTIs. The annual incidence of diagnosed UTIs in the United States is greater than 10 percent for girls and 3 percent for males, and in addition to $60 \%$ of women in their lifetime, a UTI will be identified [1] [2].

Most UTIs are initiated when UPEC bacteria enter the urinary tract through the urinary meatus before ascending up the urethra and into the bladder lumen. Isolated infections of the bladder and lower urinary tract without signs or symptoms of upper urinary tract or systemic infection are referred to as "uncomplicated cystitis". Acute cystitis in non-pregnant, premenopausal patients without functional urinary tract abnormalities is classified as uncomplicated [3]. Cystitis is classified as "complicated cystitis" in pregnant or immunocompromised patients and patients with functional urinary tract abnormalities, an indwelling catheter or a history of renal transplantation [4]. In approximately $0.34 \%$ of cases, pathogens causing cystitis ascend further through the ureters into the kidney, where they cause an infection of the renal pelvis, calices and cortex that leads to the clinical signs and symptoms of pyelonephritis [5]. If left untreated, pathogens may spread from the kidney into the bloodstream (bacteraemia), and if a concurrent systemic inflammatory response is present, this may lead to septicaemia [6]. In patients presenting to the emergency department with sepsis, $27 \%$ of all cases can be attributed to a previous urinary isolate, and are thus termed "urosepsis" [7]. In hospitalized patients, the proportion of sepsis cases attributed to UTI increases to $42 \%$ [8] [9]. There is an urgent need to develop antibiotic-sparing therapeutics that can break the vicious cycle of rUTI, antibiotic resistance and the interconnection to sepsis.

Although UPEC bacteria are the most common causative agents in both community-acquired UTI and CAUTI, the proportion of infections attributed to Gram positive bacteria and fungi are increased in CAUTI. Although reported prevalence rates differ depending on geographical location, sampling technique and diagnosis cut-offs, a recent multistate study in the United States revealed that Enterococcus faecium, Staphylococcus aureus, Klebsiella pneumoniae, Acinetobacter baumannii, Pseudomonas aeruginosa and Enterobacter species 
are the most common causative health care associated UTI pathogens after $E$. coli [10], and many of these species are commonly associated with nosocomial infections and antibiotic resistance [11] [12]. As with UPEC, the proliferation of antibiotic-resistant bacterial strains in the health-care environment has increased the cost and complexity of treating these infections [13]. Efforts to reduce the incidence of CAUTI by limiting the frequency and duration of catheter use have been successful to some extent [14] [15]. However, strict adherence to clinical guidelines to limit the screening and treatment of asymptomatic bacteriuria in the absence of symptomatic indications during catheterization, along with new treatment approaches, is needed to limit the total disease burden and proliferation of antimicrobial resistance among CAUTI strains [16] [17].

Foley catheters are useful in relieving urinary retention and controlling urinary incontinence. External microorganisms have convenient access to the bladder. The risk of contamination of the urinary tract is related to the amount of time the catheter is in operation. Many sufferers who have been catheterized for a week or much less need to prevent infection, but bacteriuria is unavoidable for the many elderly and disabled sufferers who have been catheterized for endless months or years [18] [19] [20].

The present context, of the study was divided in two parts. The first has been aimed to isolate and evaluate the clinical isolates from different sections of urinary catheter. While the second part has been aimed to evaluate the cause or route of contamination of the urinary catheter of catheterized patient and check the antimicrobial sensitivity pattern of the isolated uropathogens.

\section{Methodology}

\subsection{Study Population}

This is a prospective, multicentric study carried out at the Department of Microbiology, from January 2015 to 2018. Ethical clearance was obtained from the institutional review committee before starting the research. A total of 560 urinary catheter samples were collected from the clinically suspected patients from 12 different hospitals. As the segment wise study of urinary catheter is unique in its own and it's not done before so the pilot study was considered for the sample size over the population.

\subsection{Processing of Urinary Catheter Samples}

The urinary catheter was immediately brought to the laboratory and was processed in aseptic conditions. Clean from outside with sterile distilled water and flushed with a saline solution so that planktonic bacteria get removed. Sectioned into five appropriate parts A, B, C, D and E are as shown in Figure 1. From the catheter tip, each part was suspended into test tubes containing sterile Ringer's solution $(10 \mathrm{ml})$ separately. Sonication was performed for 5 minutes at $35 \mathrm{kHz}$ in a transonic water bath to remove and disturb the colonizing biofilm and vortex mixture was used for 2 minutes. 


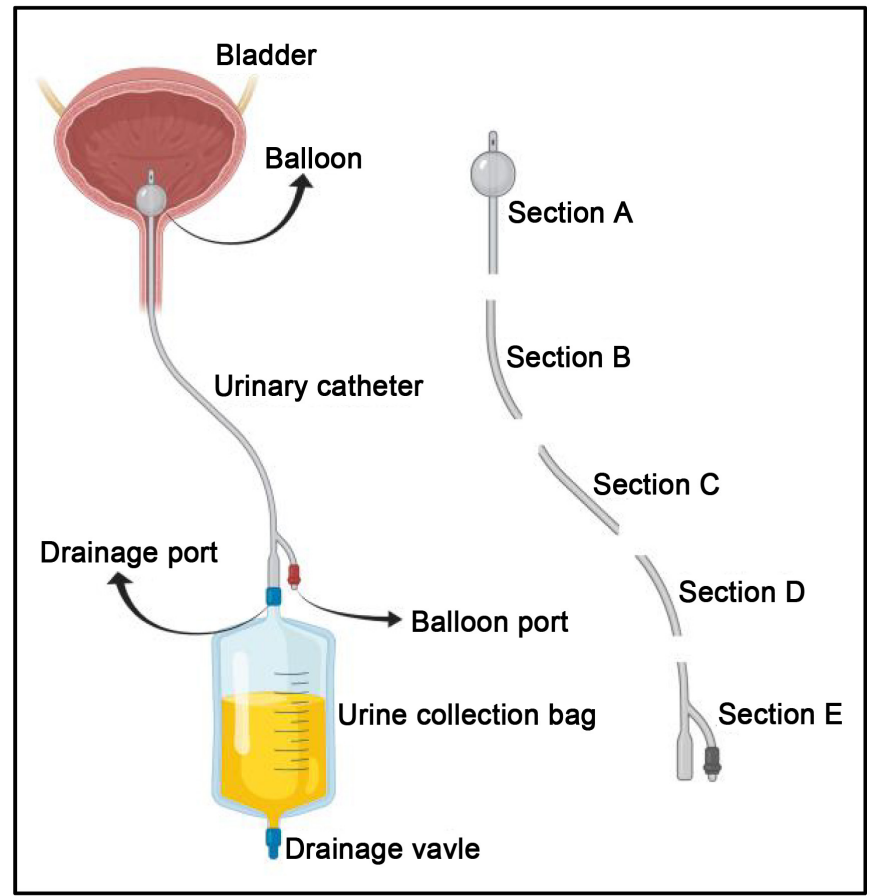

Figure 1. Urinary catheter placed in the urinary bladder and different sections of catheter.

\subsection{Isolation of Uropathogens}

Resulting cell suspensions were cultured by taking a loopful of suspension on to Hichrome UTI agar plates (HiMedia Laboratories Pvt. Ltd.). The plates after streaking were incubated at $37^{\circ} \mathrm{C}$ for $24 \mathrm{~h}$. After $24 \mathrm{~h}$ incubation, the resulting colonies were identified by standard methods. All isolates were kept as stock cultures in a 5\% glycerol solution [14]. The colored colonies were observed and identified on the basis of morphological, cultural \& biochemical tests. Some bacterial cultures that could not be identified by conventional method were identified by VITEK automated identification method [21].

\subsection{Antimicrobial Susceptibility Testing}

Antimicrobial susceptibility testing was performed on Mueller-Hinton agar using disk diffusion technique according to Clinical and Laboratory Standards Institute (CLSI) guidelines [13]. The isolates were tested against the antimicrobial discs of antibiotics. (HiMedia, Mumbai, India) Amoxicillin (10 $\mu \mathrm{g})$, Cloxacillin $(5 \mu \mathrm{g})$, Erythromycin $(15 \mu \mathrm{g})$, Tetracycline $(30 \mu \mathrm{g})$, Penicillin (2 units), Gentamicin (10), Chloramphenicol (30 $\mu \mathrm{g})$, Ciprofloxacin $(5 \mu \mathrm{g})$, Nalidixic Acid (30 $\mu \mathrm{g})$, Nitrofurantoin $(300 \mu \mathrm{g})$, Levofloxacin $(5 \mu \mathrm{g})$, Imipenem $(10 \mu \mathrm{g})$, Meropenem $(10 \mu \mathrm{g})$, Ofloxacin, Tetracycline $(10 \mu \mathrm{g})$, Tobramycin $(10 \mu \mathrm{g})$, Amikacin (30 $\mu \mathrm{g})$, Gatifloxacin $(5 \mu \mathrm{g})$, Cefixime $(5 \mu \mathrm{g})$, Cefotaxime $(30 \mu \mathrm{g})$, Piperacillin (100 $\mu \mathrm{g})$, Cefpodoxime $(10 \mu \mathrm{g})$, Clindamycin $(2 \mu \mathrm{g})$, Ampicillin (10/10), Amoxyclav (20/10), Nitrofurantoin $(300 \mu \mathrm{g})$, Cefaclor $(30 \mu \mathrm{g})$, Cephoxitin $(300 \mu \mathrm{g})$, Ceftriaxone $(30 \mu \mathrm{g})$ and Ceftazidime $(30 \mu \mathrm{g})$. 


\subsection{Statistical Analysis}

The data were entered in Microsoft Excel 2013 (Microsoft Corporation Redmond, WA, USA). The Statistical analysis and interpretation has been performed using SPSS version 16 (SPSS Inc., Chicago, IL, USA). Data were expressed in terms of frequency and percentages and analyzed using chi-square tests. P-value at $<0.05$ was considered significant.

\section{Results}

In the current examination an aggregate of 560 urinary catheter tests were gathered aseptically from 12 distinct medical clinics of Amravati city, Maharashtra, India. During the assortment, patients' history was recorded alongside the span of catheterization. The catheter tests were quickly shipped to the lab for additional preparing. The handling of each example was done in five unique segments according to strategy in five areas A, B, C, D and E. Absolute of 22 distinct types of uropathogens were isolated from the urinary catheter.

Gender orientation is one of the significant variables to be considered in catheter associated urinary tract infection as there are more contaminated females catheter samples as compared to male. In the current investigation 488 catheter tests were seen as contaminated and 72 catheter of patients were with no uropathogens (Table 1).

The catheter samples collected were of patients with different age groups ranging from less than 10 to 100 years as shown in Figure 2, all the catheter samples were found with major contamination with uropathogens of the patient with almost all age group.

Table 1. Percentage and frequency of contaminated and non-contaminated urinary catheters of patients with respect to gender.

\begin{tabular}{cccc}
\hline Gender & Contaminated & Non-contaminated & Total \\
\hline Male & $188(85.45 \%)$ & $32(14.54 \%)$ & $220(39.29 \%)$ \\
Female & $300(88.23 \%)$ & $40(11.76 \%)$ & $340(60.71 \%)$ \\
Total & $488(87.14 \%)$ & $72(12.87 \%)$ & $560(100 \%)$ \\
\hline
\end{tabular}

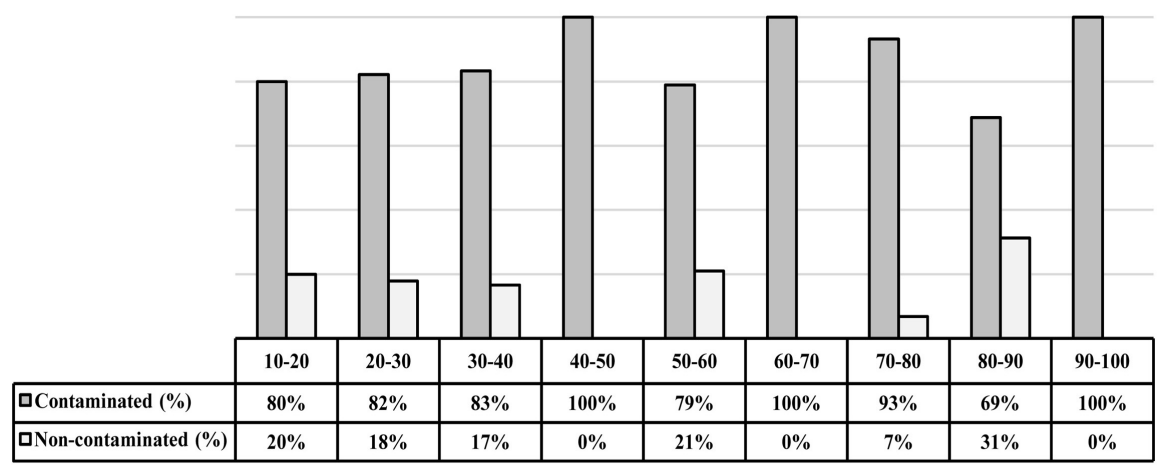

Figure 2. Percentage of contaminated and non-contaminated urinary catheter samples with age of patients. 
Duration of catheterization is one of the important factors to study the biofilm forming uropathogens isolated from urinary catheters of patients. In this study samples of different duration were collected from less than 1 day up to 40 days of catheterization as shown in Figure 3. Longer the duration, more number of bacterial and fungal loads were observed. Majority of catheter samples around $43 \%$ of samples were of 1 - 5 days and around 39\% catheter samples collected were of duration 20 - 30 days.

The samples were collected from different types of diseased patient with urinary catheters. Major catheter samples were collected from patients with Cesareansection and it was found that $88 \%$ of them were contaminated as shown in Figure 4. Almost all the catheter samples were found with contamination of uropathogens except the catheter sample of patient collected was suffering from PCNL.

The type of urinary catheter was a Foley urinary catheter. During the study it was observed that $97.8 \%$ of the urinary catheter samples collected from the patients were Two-way Foley catheter and out of that $85.7 \%$ were contaminated. In case of three-way Foley catheter only $0.6 \%$ were found to be contaminated out of total $2 \%$ as shown in Figure 5.

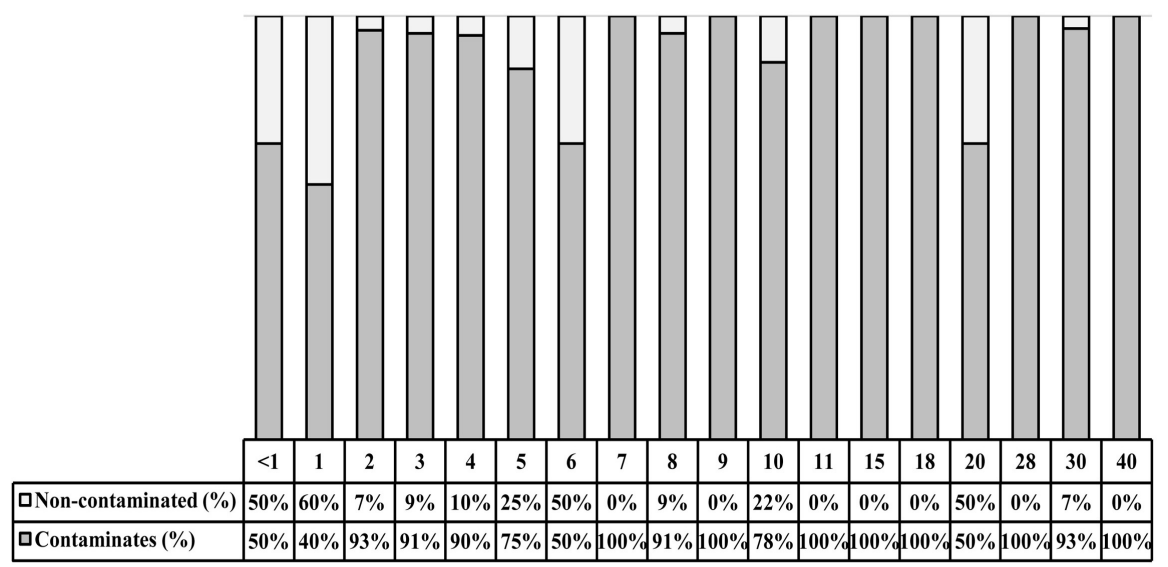

Figure 3. Percentage of contaminated and non-contaminated urinary catheter of different duration of catheterization (in Days).

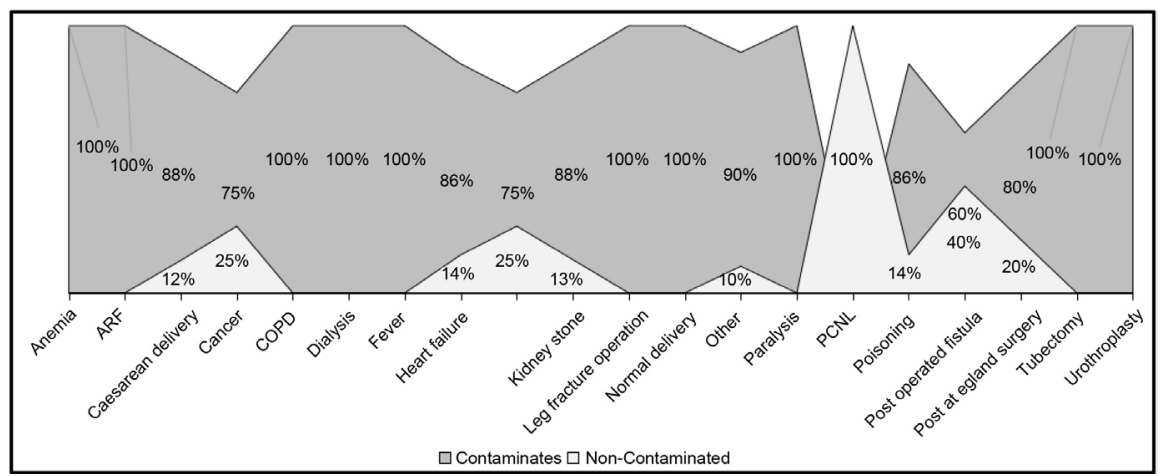

Figure 4. Percentage of contaminated and non-contaminated urinary catheter samples of disease patients. 


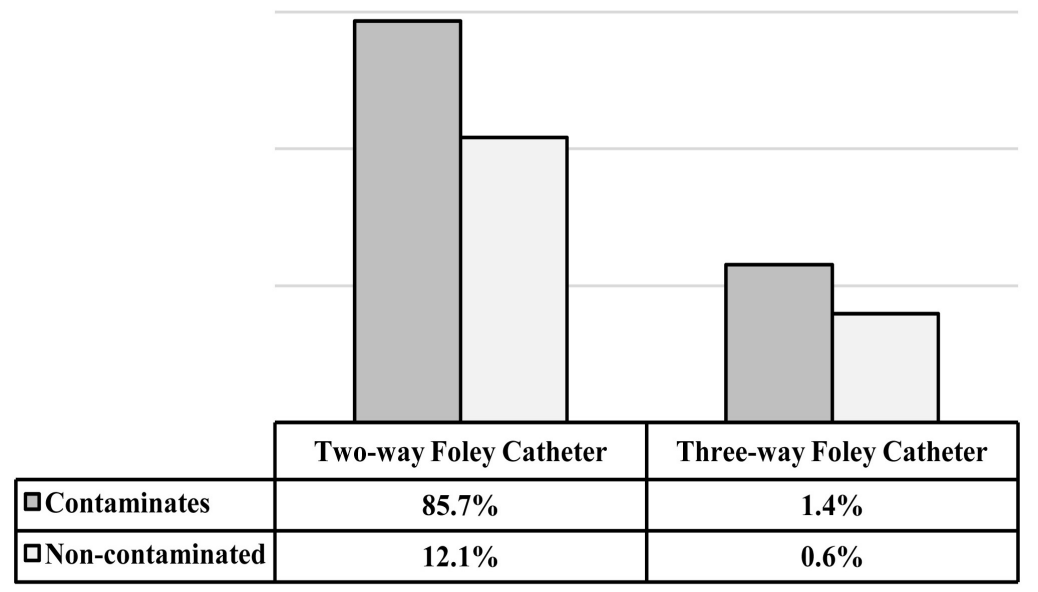

Figure 5. Type of urinary catheter collected.

Length of Foley urinary catheter were measured during the processing and as shown in Figure 6 it was found that all of them were ranged between $30-42$ centimeter length and the Foley catheter with 30 and 39 centimeter were observed with heavily contaminated with uropathogens.

\subsection{Isolation and Identification of Uropathogens}

The concept of the urethral catheter as a specific niche or reservoir for microorganisms gains support and further precision when individual species are considered. The majority of species were $P$. aeruginosa, other gram-negative bacilli, and group Staphylococci it demonstrated a fall in average concentration and prevalence $(\sim 105 \mathrm{cfu} / \mathrm{ml})$ from the indwelling to replacement catheter. Gram negative organisms were the most frequent isolates, with Pseudomonas aeruginosa (16.08\%) being the most common followed by Escherichia coli (10\%). and Klebsiella. Candida albicans accounted for almost $11 \%$ of the organisms, and was isolated in pure cultures in all the instances. In the present study, 22 different uropathogenic species were isolated as shown in Figure 7.

Urinary tract infections in catheterized patients sufferers and can happen in countless methods and pathogenic organism concentrations multiplied significantly, however, the longer a precise indwelling catheter was once in region and then fell considerably when a new catheter used to be inserted. Thus, the indwelling urethral catheter furnished the surroundings that allowed boom of positive microorganisms to concentrations greater than these in the bladder. The bladder surroundings is restrained in nutrients; thus, in order to live on and develop inside the urinary tract, uropathogens produce toxins that harm the host tissue to launch nutrients, whilst additionally supplying a area of interest for bacterial invasion and dissemination.

Uropathogens that colony the periurethral pores and skin will migrate into the bladder and contaminate through the mucoid layer that forms between the urethra's epithelial region and, in the early days of catheterization, the catheter gets contaminated. In addition, urine infection within the drainage bag may 


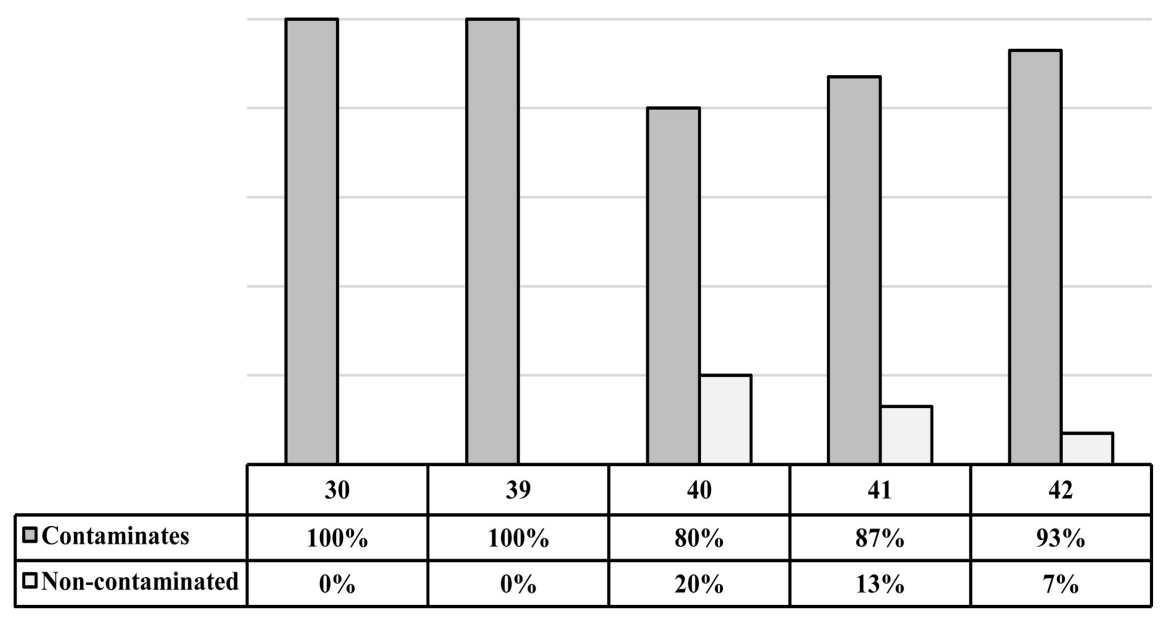

Figure 6. Percentage of contaminated and non-contaminated urinary catheters and length of collected Foley urinary catheters of patients.

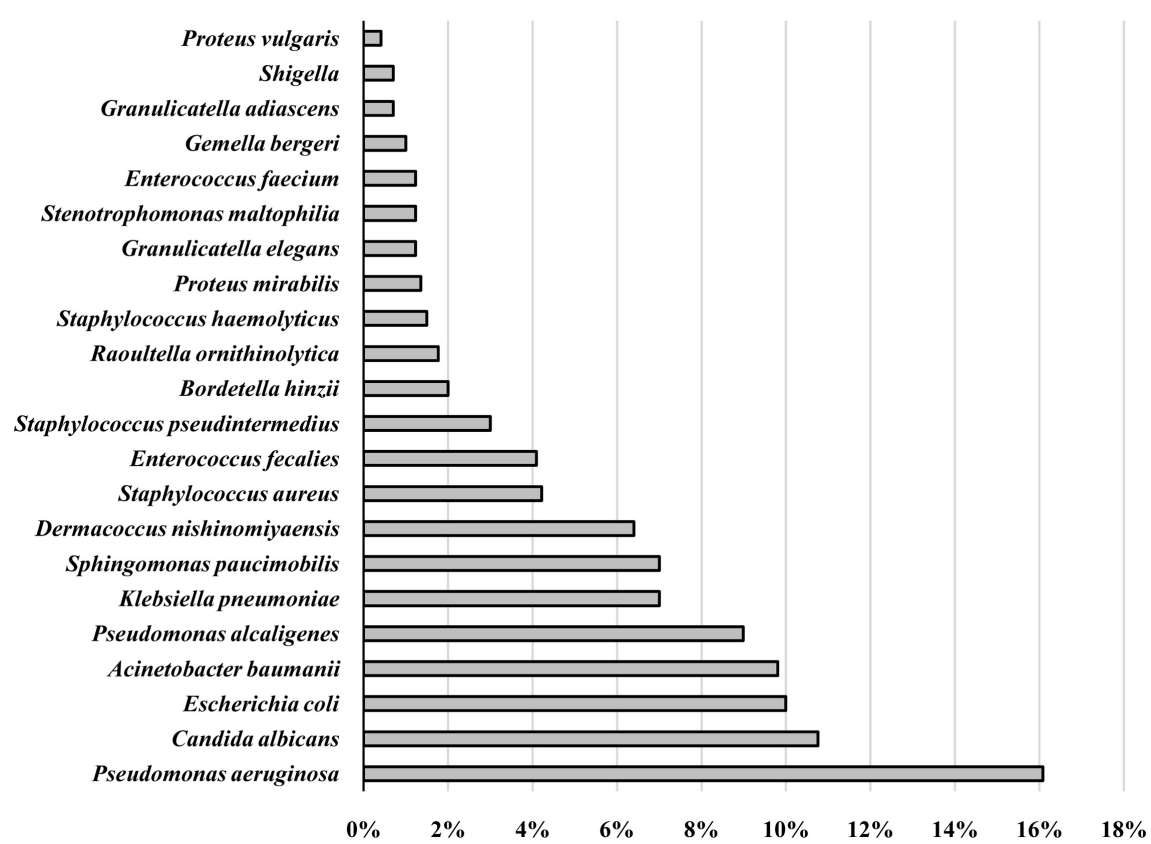

Figure 7. Percentage of Isolated uropathogens from urinary catheter samples.

cause organisms to get right through the drainage tube and therefore the catheter lumen to reach the bladder. The preliminary micro organism that is supposed to infect the tract are normally Escherichia coli. As time goes by, different species show up inside the residual bladder urine, includes Pseudomonas aeruginosa, Pseudomonas alcaligenes, Acinetobacter, Proteus mirabilis, and Klebsiella pneumoniae. Due to the fact that infections are typically asymptomatic, the micro organism that resides inside the latter levels of tract contamination is difficult to eliminate with antibiotics while the catheter is in situ, and given the possibility of encouraging antibiotic resistance, catheter-associated bacteriuria is 
usually no longer handled. Catheter modifications are often scheduled at 10 12-week intervals in patients with long-term indwelling catheters; thus, infected urine will flow via person catheters for duration of three months at a time. Thus, catheters supply desirable sites for bacterial colonization.

\subsubsection{Section A}

Majority of uropathogens were isolated from the section A (704). In which Escherichia coli was isolated highest in number. Total (112) Escherichia coli were isolated and the second most prominent uropathogen isolated was Pseudomonas aeruginosa (108). Candida species were (68). Different uropathogens were also reported in which Acinetobacter baumanii (64) highly antibiotic resistant pathogen from hospital environment, Klebsiella pneumonia (56), Pseudomonas alcaligenes (48) and Staphylococcus aureus (36) these isolated uropathogens are the normal flora of the nosocomial infections.

\subsubsection{Section B}

In section B total 576 uropathogens were detected. Pseudomonas aeruginosa (92) was found highest in number. The second most identified uropathogen were Candida species (64). Pseudomonas alcaligenes (60) was observed third most prominent uropathogen in the section B. Also Acinetobacter (56) were found in the section B. Escherichia coli were detected (48).

\subsubsection{Section C}

Catheters samples of section C 536 uropathogens were isolated and identified. In this section Pseudomonas aeruginosa was found in larger number as 88 isolates of Pseudomonas aeruginosa was isolated from section C. The second most prominent uropathogen identified was Candida species (56). Escherichia coli and Acinetobacter baumanii 52 isolates of each species were isolated. Pseudomonas alcaligenes (48), Klebsiella pneumonia (36) and Staphylococcus aureus (20) were detected.

\subsubsection{Section D}

In this section D, the highest count of Pseudomonas aeruginosa found total of 80 isolates, the second highest count of Acinetobacter baumanii (60). Candida species (56) and Pseudomonas alcaligenes (52) were found. The isolated Escherichia coli from the section D were 48. Other common nosocomial uropathogens were found in which Klebsiella pneumonia (36), Staphylococcus aureus (20) and Enterococcus fecalies (20).

\subsubsection{Section $\mathrm{E}$}

The Section E was the last section of urinary catheter has connected to the urine bag and it is present in the external environment. This section E has two openings. One was connected to urine bag to collected urine from the bladder and another one for filling the fluid to inflate the balloon. This section comes in contact with the hospital flora and it may be the reason for more contamination. In this section total 588 isolates were identified. Pseudomonas aeruginosa was 
highest in number as compare to other uropathogens. Total of 104 Pseudomonas aeruginosa species were isolated. The second contaminant in the section E was Candida species in 72 number. Acinetobacter baumanii and Pseudomonas alcaligenes were in equal count of 56 isolates. Escherichia coli were total 44 in this section. Other common uropathogens isolated from section E were Klebsiella pneumonia (32), Staphylococcus aureus (28), Enterococcus fecalies (24) and Shigella group (8).

In all the section A, B, C, D and E of the urinary catheters Pseudomonas aeruginosa was found as the most prominent uropathogen (Table 2). In the section A highest count of Escherichia coli was found which may be endogenous as the section A is inside the bladder of the catheterized patients. So there maybe possibility that the highest count of Escherichia coli from the section A was of patients flora or from inside the body. The Escherichia coli count in section $\mathrm{E}$ is the least as compare to all other sections. In section B, C, D and E the highest count of Pseudomonas aeruginosa was identified. Section E was attached to the drainage bag in contact with the hospital environment. The hospital flora is with

Table 2. Occurrence of different uropathogens in different sections of the urinary catheter.

\begin{tabular}{|c|c|c|c|c|c|}
\hline Isolated Uropathogens & $\begin{array}{c}\text { A } \\
704(23.98 \%)\end{array}$ & $\begin{array}{c}\text { B } \\
576(19.62 \%)\end{array}$ & $\begin{array}{c}C \\
536(18.26 \%)\end{array}$ & $\begin{array}{c}\mathrm{D} \\
536(18.26 \%)\end{array}$ & $\begin{array}{c}E \\
584(19.89 \%)\end{array}$ \\
\hline Escherichia coli & $112(15.90 \%)$ & $48(8.33 \%)$ & $52(9.70 \%)$ & $48(8.96 \%)$ & $44(7.53 \%)$ \\
\hline Pseudomonas alcaligenes & $48(6.81 \%)$ & $60(10.42 \%)$ & $48(8.96 \%)$ & $52(9.70 \%)$ & $56(9.59 \%)$ \\
\hline Enterococcus faecalis & $20(2.84 \%)$ & $32(5.56 \%)$ & $24(4.48 \%)$ & $20(3.73 \%)$ & $24(4.11 \%)$ \\
\hline Dermacoccus nishinomiyaensis & $36(5.11 \%)$ & $40(6.94 \%)$ & $40(7.46 \%)$ & $40(7.46 \%)$ & $32(5.48 \%)$ \\
\hline Klebsiella pneumoniae & $56(7.95 \%)$ & $36(6.25 \%)$ & $36(6.72 \%)$ & $36(6.72 \%)$ & $32(5.48 \%)$ \\
\hline Proteus mirabilis & $8(1.13 \%)$ & $20(3.47 \%)$ & $8(1.49 \%)$ & $0(0.00 \%)$ & $4(0.68 \%)$ \\
\hline Acinetobacter baumannii & $64(9.09 \%)$ & $56(9.72 \%)$ & $52(9.70 \%)$ & $60(11.19 \%)$ & $56(9.59 \%)$ \\
\hline Gemella bergeri & $12(1.70 \%)$ & $4(0.69 \%)$ & $4(0.75 \%)$ & $4(0.75 \%)$ & $4(0.68 \%)$ \\
\hline Bordetella hinzii & $12(1.70 \%)$ & $16(2.78 \%)$ & $12(2.24 \%)$ & $8(1.49 \%)$ & $8(1.37 \%)$ \\
\hline Granulicatella elegans & $12(1.70 \%)$ & $8(1.39 \%)$ & $4(0.75 \%)$ & $0(0.00 \%)$ & $12(2.05 \%)$ \\
\hline Candida albicans & $68(9.65 \%)$ & $64(11.11 \%)$ & $56(10.45 \%)$ & $56(10.45 \%)$ & $72(12.33 \%)$ \\
\hline Pseudomonas aeruginosa & $108(15.34 \%)$ & $92(15.97 \%)$ & $88(16.42 \%)$ & $80(14.93 \%)$ & $104(17.81 \%)$ \\
\hline Staphylococcus aureus & $36(5.11 \%)$ & $20(3.47 \%)$ & $20(3.73 \%)$ & $20(3.73 \%)$ & $28(4.79 \%)$ \\
\hline Stenotrophomonas maltophilia & $8(1,13 \%)$ & $4(0.69 \%)$ & $0(0.00 \%)$ & $12(2.24 \%)$ & $12(2.05 \%)$ \\
\hline Staphylococcus haemolyticus & $4(0.56 \%)$ & $4(0.69 \%)$ & $8(1.49 \%)$ & $12(2.24 \%)$ & $16(2.74 \%)$ \\
\hline Proteus vulgaris & $0(0 \%)$ & $0(0.00 \%)$ & $0(0.00 \%)$ & $8(1.49 \%)$ & $4(0.68 \%)$ \\
\hline Enterococcus faecium & $16(2.27 \%)$ & $4(0.69 \%)$ & $8(1.49 \%)$ & $4(0.75 \%)$ & $4(0.68 \%)$ \\
\hline Sphingomonas paucimobilis & $48(6.81 \%)$ & $40(6.94 \%)$ & $32(5.97 \%)$ & $36(6.72 \%)$ & $40(6.85 \%)$ \\
\hline Raoultella ornithinolytica & $12(1.70 \%)$ & $12(2.08 \%)$ & $12(2.24 \%)$ & $8(1.49 \%)$ & $8(1.37 \%)$ \\
\hline Staphylococcus pseudintermedius & $24(3.40 \%)$ & $12(2.08 \%)$ & $28(5.22 \%)$ & $12(2.24 \%)$ & $12(2.05 \%)$ \\
\hline Granulicatella adiacens & $0(0 \%)$ & $4(0.69 \%)$ & $4(0.75 \%)$ & $8(1.49 \%)$ & $4(0.68 \%)$ \\
\hline Shigella & $0(0 \%)$ & $0(0.00 \%)$ & $0(0.00 \%)$ & $12(2.24 \%)$ & $8(1.37 \%)$ \\
\hline
\end{tabular}


tremendous number of pathogens and Pseudomonas species one of the superbug present and continuously comes in contact with the section which is attached to the drainage bag. So this may be the reason of more count in section E. Also the Pseudomonas is highly motile bacterium so there was presence of Pseudomonas aeruginosa more frequently in section $\mathrm{E}$ to $\mathrm{D}, \mathrm{D}$ to $\mathrm{C}$ and $\mathrm{C}$ to $\mathrm{B}$. Very less in section A as compare to other sections. Proteus vulgaris and Shigella group were isolated only from section E and section D. Granulicatella adiacens were isolated from all other sections except section A. (Table 2). Only Candida species were isolated from fungal group in all uropathogens and highest in Section E. The present study is first of its kind that is carried out section wise to detect the contamination of indwelling urinary catheter. This can detect the route of contamination of the catheter whether it is endogenous or exogenous. So different sections were done of the catheters and were studied separately.

As the examination of the precise source of infection the isolation of uropathogens were performed from different segments. The processing of catheter samples was done section wise as explained in methodology. The varieties of uropathogens were isolated and diversity in each section were observed. Many of the uropathogens were reported as superbug and this may be the deadly cause of catheter associated infection. Total of 22 different type uropathogenic species was isolated from which some are not reported from urinary catheters before. This study reported many new uropathogens from the different sections of urinary catheter samples. Different sections were investigated and observed that the majority frequently occurring uropathogens were isolated from Section A (23.98\%) followed by section E (19.89\%). It is also reported that among sections A, B, C, D and E of the urinary catheters, Pseudomonas aeruginosa was the foremost prominent uropathogens isolated from each and each section of the urinary catheter but Escherichia coli was found more frequently from Section A.

\subsection{Antibiotic Sensitivity}

Antibiotic sensitivity pattern shows that MAR index was quite 0.2 for nearly all the uropathogens tested concluding that there's antibiotic stress on uropathogens and rate of resistance increased rapidly.

The MAR indexes of catheter associated tract pathogens have been decided with reference to 30 distinct antibiotics. The values for all the strains have been above 0.2 suggesting their beginning from a excessive hazard source of infection where antibiotics are frequently used. Emergence of MAR pathogenic strains of Pseudomonas aeruginosa and Escherichia coli indicated viable nosocomial contamination inside the clinic environment. Most catheter related tract infections are polymicrobial. The twin species associations chosen supported their occurrence in scientific settings have been Klebsiella pneumoniae, Escherichia coli, Enterococcus fecalies and Proteus mirabilis (Table 3). All species developed single species in artificial urine. The findings disclose bacterial interactions between uropathogens in dual-species ranged from affecting preliminary illness to 
Table 3. Multiple antibiotic resistance index of uropathogens.

\begin{tabular}{|c|c|c|c|c|c|c|c|c|c|c|c|}
\hline MAR Index & 0 & 0.1 & 0.2 & 0.3 & 0.4 & 0.5 & 0.6 & 0.7 & 0.8 & 0.9 & 1 \\
\hline Escherichia coli $(n=304)$ & $0 \%$ & $1 \%$ & $1 \%$ & $7 \%$ & $16 \%$ & $28 \%$ & $23 \%$ & $19 \%$ & $4 \%$ & $1 \%$ & $0 \%$ \\
\hline Enterococcus faecalis $(n=120)$ & $0 \%$ & $1 \%$ & $11 \%$ & $22 \%$ & $13 \%$ & $9 \%$ & $20 \%$ & $22 \%$ & $3 \%$ & $0 \%$ & $0 \%$ \\
\hline Pseudomonas alcaligenes (264) & $0 \%$ & $0 \%$ & $2 \%$ & $10 \%$ & $5 \%$ & $3 \%$ & $17 \%$ & $42 \%$ & $19 \%$ & $2 \%$ & $0 \%$ \\
\hline Dermacoccus nishinomiyaensis $(n=188)$ & $0 \%$ & $0 \%$ & $3 \%$ & $13 \%$ & $18 \%$ & $10 \%$ & $15 \%$ & $25 \%$ & $14 \%$ & $3 \%$ & $0 \%$ \\
\hline Klebsiella pneumoniae $(\mathrm{n}=196)$ & $0 \%$ & $3 \%$ & $14 \%$ & $23 \%$ & $20 \%$ & $20 \%$ & $15 \%$ & $5 \%$ & $0 \%$ & $0 \%$ & $0 \%$ \\
\hline Proteus mirabilis $(n=40)$ & $0 \%$ & $0 \%$ & $20 \%$ & $23 \%$ & $43 \%$ & $10 \%$ & $5 \%$ & $0 \%$ & $0 \%$ & $0 \%$ & $0 \%$ \\
\hline Acinetobacter baumannii $(n=288)$ & $0 \%$ & $17 \%$ & $24 \%$ & $29 \%$ & $16 \%$ & $10 \%$ & $3 \%$ & $1 \%$ & $0 \%$ & $0 \%$ & $0 \%$ \\
\hline Gemella bergeri $(\mathrm{n}=28)$ & $0 \%$ & $0 \%$ & $0 \%$ & $4 \%$ & $39 \%$ & $39 \%$ & $18 \%$ & $0 \%$ & $0 \%$ & $0 \%$ & $0 \%$ \\
\hline Granulicatella elegans (36) & $0 \%$ & $3 \%$ & $31 \%$ & $22 \%$ & $0 \%$ & $11 \%$ & $28 \%$ & $6 \%$ & $0 \%$ & $0 \%$ & $0 \%$ \\
\hline Pseudomonas aeruginosa (472) & $0 \%$ & $6 \%$ & $40 \%$ & $33 \%$ & $15 \%$ & $5 \%$ & $0 \%$ & $0 \%$ & $0 \%$ & $0 \%$ & $0 \%$ \\
\hline Staphylococcus aureus (124) & $2 \%$ & $14 \%$ & $34 \%$ & $27 \%$ & $15 \%$ & $7 \%$ & $2 \%$ & $0 \%$ & $0 \%$ & $0 \%$ & $0 \%$ \\
\hline Stenotrophomonas maltophilia $(n=40)$ & $0 \%$ & $0 \%$ & $28 \%$ & $63 \%$ & $10 \%$ & $0 \%$ & $0 \%$ & $0 \%$ & $0 \%$ & $0 \%$ & $0 \%$ \\
\hline Staphylococcus haemolyticus $(\mathrm{n}=44)$ & $0 \%$ & $0 \%$ & $5 \%$ & $30 \%$ & $57 \%$ & $9 \%$ & $0 \%$ & $0 \%$ & $0 \%$ & $0 \%$ & $0 \%$ \\
\hline Proteus vulgaris $(\mathrm{n}=264)$ & $0 \%$ & $17 \%$ & $50 \%$ & $25 \%$ & $8 \%$ & $0 \%$ & $0 \%$ & $0 \%$ & $0 \%$ & $0 \%$ & $0 \%$ \\
\hline Enterococcus faecium $(\mathrm{n}=36)$ & $0 \%$ & $0 \%$ & $6 \%$ & $31 \%$ & $33 \%$ & $28 \%$ & $0 \%$ & $3 \%$ & $0 \%$ & $0 \%$ & $0 \%$ \\
\hline Sphingomonas paucimobilis $(\mathrm{n}=196)$ & $0 \%$ & $11 \%$ & $32 \%$ & $27 \%$ & $18 \%$ & $10 \%$ & $3 \%$ & $0 \%$ & $0 \%$ & $0 \%$ & $0 \%$ \\
\hline Raoultella ornithinolytica $(\mathrm{n}=52)$ & $0 \%$ & $0 \%$ & $4 \%$ & $23 \%$ & $38 \%$ & $27 \%$ & $8 \%$ & $0 \%$ & $0 \%$ & $0 \%$ & $0 \%$ \\
\hline Granulicatella adiacens $(\mathrm{n}=20)$ & $0 \%$ & $0 \%$ & $0 \%$ & $20 \%$ & $40 \%$ & $40 \%$ & $0 \%$ & $0 \%$ & $0 \%$ & $0 \%$ & $0 \%$ \\
\hline Shigella $(\mathrm{n}=20)$ & $0 \%$ & $0 \%$ & $5 \%$ & $35 \%$ & $40 \%$ & $20 \%$ & $0 \%$ & $0 \%$ & $0 \%$ & $0 \%$ & $0 \%$ \\
\hline
\end{tabular}

outcompeting one bacterial species, counting on the identification of the companions involved. CAUTI arises thru bacterial entry into the tract by way of the urinary catheter. Risk elements for creating a CAUTI consist of extended catheterization, lady gender, older age, and diabetes.

In the present study the demographic variables considered to examine the route of contamination of urinary catheter were evaluated like duration of catheterization, disease of catheterized patient, Age, type of catheter, length of urinary catheter and rate of contaminated and non-contaminated. Table 4 shows the summary of the analysis on the basis selected demographic variables with respect to male catheterized patients and female catheterized patients (Table 4). The p-value of 0.05 or $<0.05$ signifies that the difference between the two proportions is statistically significant. The table shows that there was a statistically significant association between the duration of catheterization, type of disease, age of patient and type of catheter with respect to gender. But the $\mathrm{p}$-value is $<0.05$ in case of length of catheter and Contaminated and Non-contaminated therefore it is not statistically significant. 
Table 4. Demographic characteristics (duration, disease, age, length of catheter, type of catheter and contaminated or non-contaminated) of the patients and their association with male and female.

\begin{tabular}{|c|c|c|c|}
\hline \multicolumn{4}{|c|}{ Duration of catheterization } \\
\hline Duration (Days) & Male (\%) & Female (\%) & P-value \\
\hline$<1$ & $0(0)$ & $8(1.42)$ & $<0.001$ \\
\hline $0-5$ & $80(14.28)$ & $224(40)$ & $<0.001$ \\
\hline $6-10$ & $72(12.85)$ & $60(10.71)$ & $<0.001$ \\
\hline $11-15$ & $12(2.14)$ & $24(4.28)$ & $<0.001$ \\
\hline $16-20$ & $8(1.42)$ & $4(0.71)$ & $<0.001$ \\
\hline $26-30$ & $44(7.85)$ & $20(3.57)$ & $<0.001$ \\
\hline $36-40$ & $4(0.71)$ & $0(0)$ & $<0.001$ \\
\hline \multicolumn{4}{|c|}{ Type of Diseases } \\
\hline Acute chronic syndrome with anemia & $4(0.71)$ & $4(0.71)$ & $<0.001$ \\
\hline Acute renal failure (ARF) & $12(2.14)$ & $8(1.42)$ & $<0.001$ \\
\hline Cesarean delivery & $0(0)$ & $164(29.28)$ & $<0.001$ \\
\hline Cancer & $0(0)$ & $16(2.85)$ & $<0.001$ \\
\hline COPD & $0(0)$ & $4(0.71)$ & $<0.001$ \\
\hline Dialysis & $16(2.85)$ & $0(0)$ & $<0.001$ \\
\hline Fever & $0(0)$ & $8(1.42)$ & $<0.001$ \\
\hline Heart failure & $8(1.42)$ & $20(3.57)$ & $<0.001$ \\
\hline Hysterectomy & $0(0)$ & $16(2.85)$ & $<0.001$ \\
\hline Kidney stone & $84(15)$ & $44(7.85)$ & $<0.001$ \\
\hline Leg fracture operation & $4(0.71)$ & $0(0)$ & $<0.001$ \\
\hline Normal delivery & $0(0)$ & $4(0.71)$ & $<0.001$ \\
\hline Other & $16(2.85)$ & $24(4.28)$ & $<0.001$ \\
\hline Paralysis & $12(2.14)$ & $0(0)$ & $<0.001$ \\
\hline Percutaneous Nephrolithotomy (PCNL) & $4(0.71)$ & $0(0)$ & $<0.001$ \\
\hline Poisoning & $20(3.57)$ & $8(1.42)$ & $<0.001$ \\
\hline Post operated fistula & $8(1.42)$ & $12(2.14)$ & $<0.001$ \\
\hline Prostate gland surgery & $20(3.57)$ & $0(0)$ & $<0.001$ \\
\hline Hysterectomy & $0(0)$ & $4(0.71)$ & $<0.001$ \\
\hline Urethroplasty & $12(2.14)$ & $4(0.71)$ & $<0.001$ \\
\hline \multicolumn{4}{|c|}{ Age (Years) } \\
\hline $10-20$ & $11(1.96)$ & $4(0.71)$ & $<0.001$ \\
\hline $21-30$ & $29(5.17)$ & $111(19.82)$ & $<0.001$ \\
\hline $31-40$ & $28(5)$ & $92(16.42)$ & $<0.001$ \\
\hline $41-50$ & $31(5.53)$ & $33(5.89)$ & $<0.001$ \\
\hline $51-60$ & $28(5)$ & $48(8.57)$ & $<0.001$ \\
\hline $61-70$ & $48(8.57)$ & $32(5.71)$ & $<0.001$ \\
\hline $71-80$ & $32(5.71)$ & $12(2.14)$ & $<0.001$ \\
\hline $81-90$ & $8(1.42)$ & $8(1.42)$ & $<0.001$ \\
\hline $91-100$ & $4(0.71)$ & $0(0)$ & $<0.001$ \\
\hline
\end{tabular}


Continued

\begin{tabular}{lccc}
\hline \multicolumn{4}{c}{ Type of catheter } \\
\hline $\begin{array}{l}\text { Two-way Foley Catheter } \\
\text { Three-way Foley Catheter }\end{array}$ & $212(37.85)$ & $336(60)$ & 0.05 \\
\hline \multicolumn{4}{c}{ Total length of Urinary catheter (Centimeter) } \\
\hline 30 & $4(0.71)$ & $4(0.71)$ & 0.05 \\
39 & $4(0.71)$ & $4(0.71)$ & $>0.05$ \\
40 & $56(10)$ & $104(18.57)$ & $>0.05$ \\
41 & $96(17.14)$ & $120(21.42)$ & $>0.05$ \\
42 & $60(10.71)$ & $108(19.28)$ & $>0.05$ \\
\hline \multicolumn{5}{c}{ Contaminated and Non-contaminated urinary catheters } \\
\hline Non-Contaminated & $32(5.71)$ & $40(7.14)$ & $>0.05$ \\
Contaminated & $188(33.57)$ & $300(53.57)$ & $>0.05$ \\
\hline
\end{tabular}

\section{Discussion}

Most UTIs are started sooner than ascending up the urethra and into the bladder lumen when the UPEC micro organism reaches the urinary tract via the urinary meatus. In addition to signs and symptoms or signs and symptoms of higher urinary tract or systemic contamination, isolated bladder infections and urinary tract reduction are referred to as "uncomplicated cystitis" or "simple cystitis." In non-pregnant, premenopausal patients without functional urinary tract anomalies, acute cystitis is graded as uncomplicated [3]. In pregnant or immunocompromised patients and patients with useful urinary tract anomalies, an indwelling catheter or a history of renal transplantation, cystitis is classified as "complicated cystitis" [4]. Pathogens inflicting cystitis grow similarly through the ureters into the kidney in about 0.34 percent of cases, the place they cause contamination of the renal pelvis, calices and cortex that contributes to medical symptoms and signs of pyelonephritis [5]. Pathogens can additionally spread from the kidney into the bloodstream (bacteraemia) if left untreated, and if there is a corresponding systemic inflammatory reaction, this can also lead to septicaemia [6]. For patients imparting sepsis to the emergency branch, 27 percent of all cases may be linked to a prior urinary isolate, and are thus referred to as "urosepsis" [7]. The proportion of cases of sepsis due to UTI in hospitalised patients will rise to 42 percent [8]. Antibiotic-sparing therapeutics that can ruin the vicious cycle of rUTI, antibiotic resistance and interconnection to sepsis need to be improved urgently.

Although UPEC uropathogens are the most frequent causative dealers in any UTI and CAUTI acquired by the population, CAUTI extends the percentage of infections attributed to Gram positive uropathogens and fungi. Although the above incidence rates differ depending on geographical location, sampling method and diagnosis cut-offs, Enterococcus faecium, Staphylococcus aureus, Klebsiella pneumoniae, Acinetobacter baumanii, Staphylococcus aureus, Acine- 
tobacter baumanii, Pseudomonas, and Pseudomonas have been reported in the United States., and many of these species are frequently related with nosocomial infections and antibiotic resistance [11] [12] As with UPEC, the prevalence of antibiotic-resistant bacterial traces has increased the expense and difficulty of treating these infections in the health-care environment [13]. Efforts to reduce the incidence of CAUTI by restricting the frequency and duration of use of the catheter have to some degree been successful [15]. However, in the absence of symptomatic symptoms, strict adherence to medical guidelines to limit the screening and remedy of asymptomatic bacteriuria in the absence of symptomatic symptoms throughout catheterization, alongside with new cure approaches, is wished to restrict the whole disorder burden and proliferation of antimicrobial resistance amongst CAUTI strains [16].

\section{Conclusion}

During the study, varieties of uropathogens were isolated and diversity in each section was observed. Many of the uropathogens were reported as superbug and this may be the deadly cause of catheter associated infection. The present study reported different uropathogens from the each sections of urinary catheter of patient. Escherichia coli may be endogenous as the A section is inside the urinary bladder of the catheterized patients. So there may be a possibility that it may be from patients' flora or from inside the body. The Escherichia coli is found to be the second most dominant uropathogens in the present study but the count of Escherichia coli in section E is the lesser as compared to sections A, $\mathrm{B}, \mathrm{C}$ and $\mathrm{D}$. The E section was connected to the drainage bag and it is in contact with the hospital environment or the outer environment. Also the hospital flora is with a tremendous number of pathogens and Pseudomonas species is highly mobile and the most prominent superbug present continuously in the outer environment, which shows that it might be the reason for the presence of the greatest count of Pseudomonas species in the lower section of the urinary catheter. Major groups of Candida albicans were isolated from section E. Length of catheter is also one of the demographic factor in the present study and this shows that the shorter the length of catheter, the more probability of contamination.

The present study is first of its kind that is carried out section wise to detect the main influential factor for contamination of indwelling urinary catheters. This can detect the route of contamination of the catheter whether it is endogenous or exogenous.

\section{Conflicts of Interest}

There is no conflict of interest.

\section{Funding}

The research was supported by University Grant Commission New Delhi India 
under Major Research Project with reference No. [F. No. 40-472/2014(SR)].

\section{Ethics Approval}

Ethical clearance was obtained from the institutional ethical committee.

\section{Availability of Data and Material}

Not applicable.

\section{Code Availability}

Not applicable.

\section{Authors' Contributions}

All authors contributed to the study conception and design. Material preparation, data collection and analysis were performed by Anjali A. Tiwari, Niraj Ghnawate and Yogesh Khalshinge. The first draft of the manuscript was written by Anjali A. Tiwari and all authors commented on previous versions of the manuscript. All authors read and approved the final manuscript.

\section{References}

[1] Foxman, B., Barlow, R., D’Arcy, H., Gillespie, B. and Sobel, J.D. (2000) Urinary Tract Infection: Self-Reported Incidence and Associated Costs. Annals of Epidemiology, 10, 509-515. https://doi.org/10.1016/S1047-2797(00)00072-7

[2] Foxman, B. and Brown, P. (2003) Epidemiology of Urinary Tract Infections: Transmission and Risk Factors, Incidence, and Costs. Infectious Disease Clinics of North America, 17, 227-241. https://doi.org/10.1016/S0891-5520(03)00005-9

[3] Hooton, T.M. and Gupta, K. (2018) Acute Simple Cystitis in Women.

[4] Hooton, T.M. (2012) Clinical Practice. Uncomplicated Urinary Tract Infection. New England Journal of Medicine, 366, 1028-1037. https://doi.org/10.1056/NEJMcp1104429

[5] Katchman, E.A., et al. (2005) Three-Day vs Longer Duration of Antibiotic Treatment for Cystitis in Women: Systematic Review and Meta-Analysis. The American Journal of Medicine, 118, 1196-1207. https://doi.org/10.1016/j.amjmed.2005.02.005

[6] Moyo, S.J., Aboud, S., Kasubi, M., Lyamuya, E.F. and Maselle, S.Y. (2010) Antimicrobial Resistance among Producers and Non-Producers of Extended Spectrum Beta-Lactamases in Urinary Isolates at a Tertiary Hospital in Tanzania. BMC Research Notes, 3, 348. https://doi.org/10.1186/1756-0500-3-348

[7] Seymour, C.W., et al. (2017) Time to Treatment and Mortality during Mandated Emergency Care for Sepsis. New England Journal of Medicine, 376, 2235-2244. https://doi.org/10.1056/NEJMoa1703058

[8] Hatfield, K.M., et al. (2018) Assessing Variability in Hospital-Level Mortality among U.S. Medicare Beneficiaries with Hospitalizations for Severe Sepsis and Septic Shock. Critical Care Medicine, 46, 1753-1760. https://doi.org/10.1097/CCM.0000000000003324

[9] Flores-Mireles, A.L., Walker, J.N., Caparon, M. and Hultgren, S.J. (2015) Urinary Tract Infections: Epidemiology, Mechanisms of Infection and Treatment Options. 
Nature Reviews Microbiology, 13, 269-284. https://doi.org/10.1038/nrmicro3432

[10] Magill, S.S., et al. (2014) Multistate Point-Prevalence Survey of Healthcare-Associated Infections. New England Journal of Medicine, 370, 1198-1208.

https://doi.org/10.1056/NEJMoa1306801

[11] Rice, L.B. (2008) Federal Funding for the Study of Antimicrobial Resistance in Nosocomial Pathogens: No ESKAPE. The Journal of Infectious Diseases, 197, 1079-1081. https://doi.org/10.1086/533452

[12] Santajit, S. and Indrawattana, N. (2016) Mechanisms of Antimicrobial Resistance in ESKAPE Pathogens. BioMed Research International, 2016, Article ID: 2475067. https://doi.org/10.1155/2016/2475067

[13] Weiner, L.M., et al. (2016) Antimicrobial-Resistant Pathogens Associated with Healthcare-Associated Infections: Summary of Data Reported to the National Healthcare Safety Network at the Centers for Disease Control and Prevention, 2011-2014. Infection Control \& Hospital Epidemiology, 37, 1288-1301. https://doi.org/10.1017/ice.2016.174

[14] Macleod, S.M. and Stickler, D.J. (2007) Species Interactions in Mixed-Community Crystalline Biofilms on urinary Catheters. Journal of Medical Microbiology, 1549-1557.

[15] Centers for Disease Control and Prevention (2016) 2014 HAI Progress Report.

[16] Daniels, K.R., Lee, G.C. and Frei, C.R. (2014) Trends in Catheter-Associated Urinary Tract Infections among a National Cohort of Hospitalized Adults, 2001-2010. American Journal of Infection Control, 42, 17-22. https://doi.org/10.1016/j.ajic.2013.06.026

[17] Meddings, J., Rogers, M.A., Macy, M. and Saint, S. (2010) Systematic Review and Meta-Analysis: Reminder Systems to Reduce Catheter-Associated Urinary Tract Infections and Urinary Catheter Use in Hospitalized Patients. Clinical Infectious Diseases, 51, 550-560. https://doi.org/10.1086/655133

[18] Warren, J.W. (1991) The Catheter and Urinary Tract Infection. Medical Clinics of North America, 75, 481-493. https://doi.org/10.1016/S0025-7125(16)30465-5

[19] Kunin, C.M. (1997) Care of the Urinary Catheter. In: Kunin, C.M., Ed., Urinary Tract Infections. Detection, Prevention and Management, 5th Edition, Williams and Wilkins, Baltimore, 226-278.

[20] Stickler, D.J. (2008) Bacterial Biofilms in Patients with Indwelling Urinary Catheters. Nature Clinical Practice Urology, 5, 598-608. https://doi.org/10.1038/ncpuro1231

[21] Pincus, D.H. (2007) New Vitek 2 Colorimetric GN Card for Identification of Gram-Negative Nonfermentative Bacilli. Journal of Clinical Microbiology, 45, 4094-4095. https://doi.org/10.1128/JCM.01457-07 